\title{
The Effect of Cabergoline on Sleep, Periodic Leg Movements in Sleep, and Early Morning Motor Function in Patients with Parkinson's Disease
}

\author{
Birgit Högl',2, Andreas Rothdach', Thomas C Wetter' and Claudia Trenkwalder*,1,3 \\ 'Max Planck Institute of Psychiatry, Department of Neurology, Munich, Germany; ${ }^{2}$ Department of Neurology, University of Innsbruck, Innsbruck, \\ Austria; ${ }^{3}$ Department of Clinical Neurophysiology, University of Göttingen, Göttingen, Germany
}

To investigate the effect of the dopamine D2 and DI receptor agonist cabergoline on sleep, periodic leg movements (PLMs) in sleep, and early morning motor performance in patients with Parkinson's disease (PD). It was hypothesized that cabergoline had long-lasting beneficial effects on sleep and PLMs in sleep in patients with PD, after a single evening intake. A total of I 5 patients with idiopathic PD underwent two nights of polysomnography and motor tests (UPDRS, tapping test) before and after 6-8 weeks of treatment with cabergoline (dosage: 3-6 mg/day). Additionally, patients completed a subjective sleep visual analog scale (VAS) before and during cabergoline treatment. Compared to baseline values, treatment with cabergoline did not change sleep efficiency or the amount of stage I and stage 2 sleep. The number of awakenings $(22.4 \pm 10.1$ vs $32.5 \pm 13.3, p<0.05)$ and stage shifts ( I I9 \pm 42 vs 148 $\pm 46, p<0.05)$ were increased during treatment with cabergoline, and PLMs in sleep were reduced (PLM index $34.9 \pm 44.9$ vs $6.7 \pm 4.2$ per hour, $p<0.05)$. Cabergoline significantly improved early morning motor function, and in spite of increased phase shifts and awakenings, patients felt significantly more refreshed in the morning during cabergoline therapy. Cabergoline slightly fragmented sleep, without altering its total amount. The functional significance of this finding is uncertain. The subjective quality of sleep improved, and periodic limb movements in sleep decreased.

Neuropsychopharmacology (2003) 28, I866-1870, advance online publication, 16 July 2003; doi: I0.1038/sj.npp. 1300250

Keywords: sleep; Parkinson's disease; polysomnography; periodic limb movements (PLMs); dopamine agonist; nocturnal akinesia; insomnia; cabergoline

\section{INTRODUCTION}

Sleep disturbance is present in more than $60 \%$ of patients with Parkinson's disease (PD) (Lees et al, 1988; Trenkwalder, 1998; Tandberg et al, 1998). Even $98 \%$ of PD patients have experienced at least one symptom that was disruptive to their sleep (Lees et al, 1988) during the duration of their disease. Many factors are thought to contribute to this sleep disruption, such as the diseaserelated dopaminergic deficit, dopaminergic and nondopaminergic drug therapy, and motor symptoms, for example, nocturnal akinesia, tremor, or periodic limb movements in sleep (PLMS) (Poewe and Högl, 2000).

*Correspondence: Dr C Trenkwalder, Department of Clinical Neurophysiology, Georg-August-University Göttingen, Robert-KochStr. 40, 37075 Göttingen, Germany, Tel: + 49 55I 39 8453, Fax: + 49 55। 39 |265।, E-mail: ctrenkw@gwdg.de

Received 27 September 2002; revised 03 January 2003; accepted 13 January 2003

Online publication: 27 May 2003 at http://www.acnp.org/citations/ Npp052702357/default.pdf
Recent studies show increasing evidence that the disturbance of sleep and wakefulness in PD is most intimately related to the disease itself and does not exclusively represent a secondary phenomenon (Poewe and Högl, 2000; Rye and Jankovic, 2002). This primary effect of PD on sleep may be linked to an early recognized (Wauquier, 1995), although only recently fully appreciated, role of dopamine in modulating sleep wake state (Rye and Jankovic, 2002) and a newly described mesothalamic dopaminergic pathway (Freeman et al, 2001).

PLMS are trains of repetitive, quite stereotyped movements in sleep frequently involving the lower limbs and leading to myoclonic or dystonic muscle activity (Montplaisir et al, 2000; Provini et al, 2001). PLMS occur frequently in PD patients (Wetter et al, 2000) and are also associated with sleep disorders involving a dopaminergic impairment (Montplaisir et al, 2000).

Among the various factors, nocturnal akinesia is considered to play a major role in the pathomechanism of sleep disturbance in patients with PD (Lees et al, 1988; Trenkwalder, 1998). It is well known that nocturnal akinesia and sleep disturbance in PD can be improved by optimizing 
dopaminergic therapy. However, treatment of nocturnal symptoms with levodopa is complicated by its short halflife, which is only in part resolved by using sustained release formulations (Chouza et al, 1990; Pahwa et al, 1993). Distinct from levodopa and other dopamine agonists, the elimination half-life of cabergoline, a dopamine D2 and D1 receptor agonist, is longer than $65 \mathrm{~h}$ (Fariello, 1998). In spite of once-daily dosing, stable plasma levels of cabergoline are maintained (Lera et al, 1993). We hypothesized that cabergoline would be, in particular, appropriate for improving akinesia-induced sleep disturbance. The present study was undertaken to evaluate the effect of monotherapy or add-on therapy with cabergoline on sleep, periodic leg movements in sleep, and morning motor performance in patients with PD.

\section{METHODS}

\section{Design}

In this open clinical trial, we evaluated the effect of monoor add-on therapy with cabergoline on sleep variables (analyzed by a blind rater, see below) and morning motor function. The patients were evaluated before beginning cabergoline therapy and 6-8 weeks later, when the optimal cabergoline dose with respect to motor performance had been reached. All patients underwent polysomnography and morning motor evaluations and completed a subjective sleep visual analog scale (VAS) before and while on cabergoline therapy.

\section{Patients}

Patients with idiopathic PD were consecutively recruited from the PD outpatient clinic. They were eligible, if they were not receiving therapy for $\mathrm{PD}$ (de novo patients) or if pretreatment with dopaminergic agents was unsatisfactory with respect to motor symptoms. Patients with suspected multiple system atrophy or other clinically atypical PD, dementia, or drug-induced hallucinations were excluded from the study.

A total of 17 patients with idiopathic PD were included. Two dropped out of the study, as they did not agree to a second polysomnography during cabergoline therapy. Despite leaving the study, both patients wished to continue treatment with cabergoline.

In all, 15 patients completed the protocol (eight males, seven females, mean age $63.9 \pm 9.5$ years, mean duration of PD symptoms $4.5 \pm 3.3$ years). Hoehn and Yahr stage (Fahn et al, 1987) was 2.5 (1-3) (median, range). Six patients were de novo, and nine had been pretreated with levodopa (200$800 \mathrm{mg} /$ day).

Drug titration was started with $1 \mathrm{mg}$ of cabergoline in the evening, and weekly increased by $1 \mathrm{mg}$ until a sufficient control of motor symptoms was reached according to the patients' opinion. In parallel, the daily levodopa dose was reduced according to the extent possible without worsening of motor state.

Polysomnography was performed with a Schwarzer ED24 polysomnograph with paper printouts (Schwarzer Inc., Munich, Germany) using a standard polysomnography montage (Rechtschaffen and Kales, 1968) including C3,
C4, A1, and A2, electro-oculography and chin-electromyography. Additionally, EMG of both tibialis anterior muscles was recorded for leg movements, and recording of ECG and respiratory variables (oro-nasal airflow, tracheal microphone, thoracal and abdominal respiratory effort, and oxygen saturation) was performed. In the baseline and treatment condition, two consecutive nights were recorded. The first night was considered an adaptation night, and only the results from the second night were used for analysis. Sleep was scored according to standard criteria (Rechtschaffen and Kales, 1968; ASDA, 1992). REM sleep without atonia and REM sleep behavior disorder were defined according to proposed criteria (Lapierre and Montplaisir, 1992; Mahowald and Schenck, 1995). Amounts of wakefulness and sleep stages were calculated as percentage of sleep period time. Sleep stage scoring was performed by an experienced technician blind to the patients' condition and treatment and was supervised by one of the authors, who also evaluated the studies for presence of REM sleep without atonia and sleep-disordered breathing (TCW).

Periodic leg movements (PLM) in sleep were recorded and counted by a blind rater according to the ASDA taskforce recommendations for PLM (ASDA, 1993). PLM counts are given as indices (PLM/h). The minimum index calculated was 5 per hour (PLM indices below 5 were calculated as $<5)$.

\section{UPDRS Motor Scale and other Subtests, Tapping Tests}

In addition to sleep evaluations, the effect of cabergoline on morning motor performance at baseline was monitored with UPDRS III (Fahn et $a l$, 1987) and with additional tapping tests at 0800 in the morning before intake of any morning medication. The 1-min tapping rate was calculated as the number of times the patient could tap on two $4 \times 4 \mathrm{~cm}$ marks placed on a board $30 \mathrm{~cm}$ apart during $1 \mathrm{~min}$. Tapping rates from both hands were added. All motor tests were performed by a single rater (AR). The last previous evening dose of medication was allowed at 1800. Also, the mentation behavior and mood, activities of daily living, and side effects of therapy subscales of the UPDRS (Fahn et al, 1987) were recorded at baseline and while on cabergoline therapy.

\section{Subjective Sleep Perception}

The patients also gave subjective estimates of sleep on selfadministered VASs with four items (Table 2). The VAS was completed on the morning of the second study day. Each answer could be graded from 1 (worst) to 10 (best).

\section{Side Effects}

During the entire study period, there were no clinically relevant side effects to be observed except mild nausea without the necessity to administer domperidon in any patient. Blood pressure had not been systematically controlled, but no patient reported signs of orthostatic hypotension. One patient had intermittent diarrhea during cabergoline therapy. One patient suffered from psychosis shortly after the study period (dosage cabergoline $4 \mathrm{mg}$, LDOPA $400 \mathrm{mg}$ ) and cabergoline had been stopped immedi- 
ately and clozapine had been started until the psychosis remitted.

\section{RESULTS}

The final cabergoline dose (leading to optimum motor performance during the day) ranged between 3 and $6 \mathrm{mg}$ (median: $5 \mathrm{mg}$ ). After addition of cabergoline, the levodopa dose was reduced in five patients, but in no patient was it discontinued (range of reduction: $50-200 \mathrm{mg} /$ day). One patient each had additional selegiline, tolcapone, and budipine, which were all maintained after addition of cabergoline.

\section{Polysomnography and PLMs}

Total amount of sleep, wakefulness after sleep onset, sleep and REM sleep latency, and relative amounts of sleep stages were not different before and during treatment with cabergoline (see Table 1). Significant increases in stage shifts, awakenings, and movement time were found with cabergoline therapy (Table 1). Increased tonic or phasic muscle activity during REM sleep (corresponding to REM without atonia) was present in seven subjects and was qualitatively not altered by cabergoline therapy. Four patients had mild or moderate sleep disordered breathing with respiratory distress indices between 10 and 20/h.

A significant decrease in PLMs was found during cabergoline treatment compared to baseline (PLM index $34.9 \pm 44.9$ vs $6.7 \pm 4.2$ while on cabergoline treatment, $p=0.022)$ (see Table 1$)$.

\section{Motor Tests}

UPDRS activity of daily living (part II), motor part (part III) and tapping tests were significantly improved on cabergoline therapy compared to baseline (see Table 2).

\section{Sleep Perception}

The results of the subjective sleep scale are given in Table 2 .

\section{DISCUSSION}

In this prospective, open study, cabergoline treatment increased arousals, stage shifts, and awakenings, although quantitative polygraphic measures of sleep were maintained and subjective measures of sleep quality even improved. Cabergoline significantly reduced PLMS and improved early morning akinesia.

Polysomnographic recordings at baseline presented with a low sleep efficiency and high sleep fragmentation (expressed in increased stage shifts and arousals), which is typical for PD (Askenasy, 1993). With cabergoline therapy, we found a trend towards increased stage 1 and stage 2 sleep. However, epoch assignment to conventional Rechtschaffen and Kales sleep stages has a limited reliability in PD (Bliwise et al, 2000), a drawback, that can possibly be resolved by more sophisticated forms of analyses (Brunner et al, 2002). When non-REM sleep stages one to four were summed up as has been proposed for PD (Bliwise et al, 2000), there was still no difference in the amount before and during cabergoline therapy. In contrast to other dopamine agonists (Cianchetti et al, 1980; Askenasy and Yahr, 1984), cabergoline produced no significant REM sleep suppression in our study. This might be due to the submaximal dose, or could indicate that a potential REM-sleep suppressing effect of cabergoline was partially counteracted by the improved mobility during sleep that may have facilitated the appearance of higher amounts of REM sleep.

Although sleep efficiency was not affected, polysomnography showed a significant increase in arousals and awakenings in patients on cabergoline. This may be interpreted as a reduced sleep state stability of cabergoline

Table I Sleep Variables and Periodic Leg Movements

\begin{tabular}{lccc}
\hline & Baseline & Treatment & p \\
\hline Total time of sleep (min) & $360 \pm 7 \mid$ & $373 \pm 54$ & NS \\
Sleep efficiency (\%) & $75.0 \pm 14.8$ & $77.8 \pm 11.4$ & NS \\
Sleep onset latency (min to stage 2) & $27.4 \pm 30.1$ & $14.9 \pm 13.8$ & NS \\
REM sleep latency (min) & $86.9 \pm 79.2$ & $90.7 \pm 66.9$ & NS \\
Sleep period time (SPT; min) & $445 \pm 30$ & $454 \pm 22$ & NS \\
Wakefulness after sleep onset (\% SPT) & $20.2 \pm 14.3$ & $21.2 \pm 9.6$ & NS \\
Stage I (\% SPT) & $8.0 \pm 4.2$ & $11.3 \pm 7.5$ & NS \\
Stage 2 (\% SPT) & $48.2 \pm 12.4$ & $53.4 \pm 10.9$ & NS \\
Stages 3+4 (\% SPT) & $10.9 \pm 15.9$ & $6.0 \pm 7.1$ & NS \\
REM sleep (\% SPT) & $17.6 \pm 6.6$ & $13.7 \pm 7.3$ & NS \\
Movement time (\% SPT) & $0.05 \pm 0.1$ & $0.2 \pm 0.2$ & 0.042 \\
Stage shifts (n) & $119 \pm 42$ & $148 \pm 46$ & 0.017 \\
Awakenings (n) & $22.4 \pm 10.1$ & $32.5 \pm 13.3$ & 0.013 \\
REM density & $2.31 \pm 0.81$ & $2.19 \pm 1.09$ & NS \\
Periodic leg movements index (PLM/h of sleep) & $34.9 \pm 44.9$ & $6.7 \pm 4.2$ & 0.022 \\
\hline
\end{tabular}

Polysomnographic data from 15 patients before (baseline) and during cabergoline therapy (treatment).

Definitions: sleep efficiency, total time of sleep per time in bed (TIB). TIB was fixed at 480 min; movement time

(Rechtschaffen and Kales, 1968), relative amount of 30 s epochs, which could not be scored due to artefact by gross body movement; stage shifts, number of changes in sleep state (eg between stage I and stage 2, or between stage 2 and REM), as a measure of sleep state instability; REM density, number of 3-s miniepochs of REM sleep containing one or more rapid eye movement divided through total number of 3-s miniepochs of REM sleep; statistics, statistics included MANOVA with post hoc t-tests and Bonferroni correction for multiple comparisons. 
Table 2 Effects of Cabergoline Treatment on Morning Motor Performance and Sleep

Perception

\begin{tabular}{|c|c|c|c|}
\hline & Baseline & Treatment & $\mathbf{p}$ \\
\hline \multicolumn{4}{|l|}{ Motor performance } \\
\hline UPDRS III (motor part) & $22.3 \pm 9.1$ & $14.9 \pm 9.2$ & 0.001 \\
\hline UPDRS II (activities of daily living) & $8.9 \pm 6.4$ & $4.4 \pm 3.2$ & 0.001 \\
\hline Tapping rate (taps/min) $(n=14)$ & $155.8 \pm 37.1$ & $177.4 \pm 17.2$ & 0.031 \\
\hline \multicolumn{4}{|l|}{ Sleep perception } \\
\hline How do you rate your overall sleep quality? & $5.5 \pm 3.3$ & $7.5 \pm 2.7$ & NS \\
\hline How do you rate your capacity to fall asleep? & $4.4 \pm 4.0$ & $9.1 \pm 0.7$ & 0.018 \\
\hline How do you rate your capacity to maintain sleep? & $5.6 \pm 3.4$ & $7.7 \pm 2.3$ & NS \\
\hline How refreshed do you feel now? & $5.5 \pm 3.0$ & $8.3 \pm 1.0$ & 0.02 \\
\hline
\end{tabular}

Subjective sleep perception was graded on a VAS ranging from I (worst) to 10 (best). The VAS was presented in the morning before medication intake.

Student's t-test and Wilcoxon test were used when appropriate. The UPDRS subscales I and IV did not show significant changes.

and is in line with increased spontaneous awakenings despite greatly reduced PLM in patients with restless legs syndrome undergoing treatment with the dopamine agonist pergolide (Wetter et al, 1999). As nocturnal akinesia is a major problem in patients with PD (Laihinen et al, 1987), an increase in movement time as measured in this study may indicate a treatment-induced restoration of nocturnal mobility. The increase of movement time could therefore be related to sleep improvement despite increased sleep fragmentation. In normal subjects, an inverse correlation between movement time, sleep depth, and sleep quality has been described (Blagrove et al, 1998).

Interestingly, the patients themselves found their sleep more refreshing while on cabergoline therapy. The functional significance of arousals during sleep in PD patients has not been elucidated, but the findings could point to a preponderance of nocturnal mobility over arousal counts for subjective sleep perception. However, as this was an open study, these subjective results should be interpreted with caution as a placebo effect cannot be ruled out.

The significant reduction of PLM under cabergoline treatment is in line with recent findings for cabergoline and other dopamine agonists in patients with PLM due to restless legs syndrome (Wetter et al, 1999; Montplaisir et al, 1999; Stiasny et al, 2000). As the frequency of arousals was not reduced during cabergoline therapy, this might indicate that PLMS are not the major cause for sleep fragmentation in $\mathrm{PD}$ patients.

Another finding of our study was a significant improvement in morning motor performance $24 \mathrm{~h}$ after the last cabergoline intake, attributable to the specific pharmacokinetic properties of cabergoline (Lera et al, 1993; Ahlskog et al, 1994).

Due to the relatively small number of subjects and the open-label nature of this study, the results should be considered preliminary and confirmed in a double-blind placebo-controlled trial.

We conclude that a single morning dosage of cabergoline might be specifically useful in PD patients with sleep problems, if nocturnal akinesia or PLM is suspected to contribute to the sleep disturbance.

\section{ACKNOWLEDGEMENTS}

We are grateful to F Gander PhD, Max Planck Institute of Psychiatry, Munich, for performing the statistical tests. Pharmacia supported the study and the polysomnographic recordings by a research grant.

\section{REFERENCES}

Ahlskog JE, Muenter MD, Maraganore DM, Matsumoto JY, Lieberman A, Wright KF et al (1994). Fluctuating Parkinson's disease. Treatment with the long-acting dopamine agonist cabergoline. Arch Neurol 51: 1236-1241.

Askenasy JJ (1993). Sleep in Parkinson's disease. Acta Neurol Scand 87: 167-170.

Askenasy JJ, Yahr MD (1984). Suppression of REM rebound by pergolide. J Neural Transm 59: 151-159.

Atlas Task Force of the American Sleep Disorders AssociationGuilleminault C (1993). Recording and scoring leg movements. Sleep 16: 748-759.

Atlas Task Force of the American Sleep Disorders Association (1992). EEG arousals: scoring rules and examples. Sleep 15: 173184.

Blagrove M, Owens DS, MacDonald I, Sytnik N, Tucker P, Folkard $S$ (1998). Time of day effects in, and the relationship between, sleep quality and movement. J Sleep Res 7: 233-239.

Bliwise DL, Willians ML, Irbe D, Ansari FP, Rye DB (2000). Interrater reliability for identification of REM sleep in Parkinson's disease. Sleep 23: 671-676.

Brunner H, Wetter TC, Hogl B, Yassouridis A, Trenkwalder C, Friess E (2002). Microstructure of the non-rapid eye movement sleep electroencephalogram in patients with newly diagnosed Parkinson's disease: effects of dopaminergic treatment. Movement Disord 17: 928-933.

Cianchetti C, Masala C, Mangoni A, Gessa GL (1980). Suppression of REM and delta sleep by apomorphine in man: a dopamine mimetic effect. Psychopharmacology 67: 61-65.

Chouza C, Aljanati R, Caamano JL, De Medina O, Scaramelli A, Buzo R et al (1990). Long-term treatment with Madopar HBS in parkinsonians with fluctuations. Adv Neurol 53: 519-526.

Fahn S, Elton RL, Members of the UPDRS development committee (1987). Unified Parkinson's disease rating scale. In: Fahn S, Marsden CD, Calne DB, Goldstein M (eds) Recent Development in Parkinson's Disease, Vol. 2 MacMillan Healthcare Information: Florham Park, NJ. pp 153-163; 293-304. 
Fariello RG (1998). Pharmacodynamic and pharmacokinetic features of cabergoline. Rationale for use in Parkinson's disease. Drugs (Suppl 1): 10-16.

Freeman A, Ciliax B, Bakay R, Daley J, Miller RD, Keating G et al (2001). Nigrostriatal collaterals to thalamus degenerate in parkinsonian animal models. Ann Neurol 50: 321-329.

Laihinen A, Alihanka J, Raitasuo S, Rinne UK (1987). Sleep movements and associated autonomic nervous activities in patients with Parkinson's disease. Acta Neurol Scand 76: 64-68.

Lapierre O, Montplaisir J (1992). Polysomnographic features of REM sleep behavior disorder: development of a scoring method. Neurology 42: 1371-1374.

Lees AJ, Blackburn NA, Campbell VL (1988). The nighttime problems of Parkinson's disease. Clin Neuropharmacol 11: 512-519.

Lera G, Vaamonde J, Rodriguez M, Obeso JA (1993). Cabergoline in Parkinson's disease: long-term follow-up. Neurology 43: 2587-2590.

Mahowald M, Schenck CH (1995). REM sleep behavior disorder. In: Kryger M, Roth T, Dement W (eds) Principles and Practice of Sleep Medicine, 2nd edn WB Saunders: Philadelphia. pp 574-588.

Montplaisir J, Michaud M, Denesle R, Gosselin A (2000). Periodic leg movements are not more prevalent in insomnia or hypersomnia but are specifically associated with sleep disorders involving a dopaminergic impairment. Sleep Med 1: 163-167.

Montplaisir J, Nicolas A, Denesle R, Gomez-Mancilla B (1999). Restless legs syndrome improved by pramipexole: a doubleblind randomized trial. Neurology 52: 938-943.

Pahwa R, Busenbark K, Huber SJ, Michalek D, Hubble JP, Koller WC (1993). Clinical experience with controlled-release carbidopa/levodopa in Parkinson's disease. Neurology 43: 677-681.
Poewe W, Högl B (2000). Parkinson's disease and sleep. Curr Opin Neurol 13: 423-426.

Provini F, Vetrugno R, Meletti S, Plazzi G, Solieri L, Lugaresi E et al (2001). Motor pattern of periodic limb movements during sleep. Neurology 57: 300-304.

Rechtschaffen A, Kales A (eds) (1968). A Manual of Standardized Terminology, Techniques and Scoring System for Sleep Stages of Human Subjects. Brain Information Service/Brain Research Institute, University of California: Los Angeles.

Rye DB, Jankovic J (2002). Emerging views of dopamine in modulation sleep/wake state from an unlikely source: PD. Neurology 58: 341-346.

Stiasny K, Robbecke J, Schuler P, Oertel WH (2000). Treatment of idiopathic restless legs syndrome (RLS) with the D2-agonist cabergoline - an open clinical trial. Sleep 23: 349-354.

Tandberg E, Larsen JP, Karlsen K (1998). A community-based study of sleep disorders in patients with Parkinson's disease. Movement Disord 13: 895-899.

Trenkwalder C (1998). Sleep dysfunction in Parkinson's disease. Clin Neurosci 5: 107-114.

Wauquier A (1995). Pharmacology of the catecholaminergic system. In: Kales AD (ed) The Pharmacology of Sleep. Handbook of Experimental Pharmacology, Vol. 116 Springer: Berlin, Heidelberg: New York. pp 65-85.

Wetter TC, Collado-Seidel V, Pollmächer T, Yassouridis A, Trenkwalder C (2000). Sleep and periodic leg movement patterns in drug-free patients with Parkinson's disease and multiple system atrophy. Sleep 23: 361-367.

Wetter TC, Stiasny K, Winkelmann J, Buhlinger A, Brandenburg U, Penzel $\mathrm{T}$ et al (1999). A randomized controlled study of pergolide in patients with restless legs syndrome. Neurology 52: $944-950$. 